\title{
Registration Of Sheared Images Using Phase Correlation
}

\author{
Ruben Gonzalez \\ Institute for Intelligent Systems \\ Gold Coast Campus, Griffith University \\ QLD Australia
}

\begin{abstract}
Phase Correlation is a well-known method for registration of images that are related by similarity transforms. Its extension to registration of affine transformed images is not straightforward. This paper presents a method for recovering the transformation parameters of images that have been translated, shifted, rotated and arbitrarily sheared. It is effective for recovering shearing parameters with magnitudes of up to $40 \%$ of the image size. The proposed method makes use of Phase Correlation in conjunction a Hough based technique. Theory, methodology and experimental results are presented.
\end{abstract}

Index Terms-- Affine, Fourier, Image Registration, Phase Correlation, Shearing.

\section{INTRODUCTION}

Phase Correlation is a well-known method for registration of images that are related by a similarity transformation. It is robust in the presence of noise, has constant processing time and in combination with a log-polar transform [1], can recover a wide range of global scaling, rotation and translation parameters [2]. While it is largely insensitive to localised noise and distortion it cannot handle anisotropic distortion such as produced by stretching or shearing. Registration of images related by affine transformations such as shearing is typically performed by iterative feature matching methods that are relatively slow. Furthermore phase correlation methods are still applicable in the case of images that do not contain the necessary features such as well defined corners for feature matching. There is little published work in the application of Fourier based techniques to affine transforms. This paper presents a closed-form, Fourier based solution for the recovery of parameters for shearing transforms.

Typically, frequency domain techniques have been considered unsuitable for recovering linear or affine transformation parameters [3]. Yet they have been used for estimating the spatial orientation of uniformly textured surface planes. In that case, affine parameters were obtained by comparing the frequency components, peaks or the second order moments of Fourier transforms of two patches on the textured surface $[4,5]$. The only other published method for estimating affine parameters between images in the frequency domain does so as a nonlinear minimization problem using radial projections of the squared Fourier magnitudes of the images [6]. This paper approaches the estimation of the shearing parameters as an extension of Phase Correlation. Section two presents the theoretical concepts. Section three and four discusses methodology and experimental results.

\section{THEORY}

Let $f_{1}(x, y)$ and $f_{2}(x, y)$ be two images where $[x, y] \in \Re^{2}$ and let $f_{2}$ be a version of $f_{1}$ that is sheared by factors of $z_{x}, z_{y}$ then translated by vector $\left(\mathrm{x}_{0}, \mathrm{y}_{0}\right)$, scaled by a factor $s$ and rotated by angle $\theta$, such that $f_{1}$ is related to $f_{2}$ as follows:

where:

$$
f_{2}(x, y)=f_{1}(j, k)
$$

$$
\begin{aligned}
& j=x\left(s \cos \theta+z_{x} s \sin \theta\right)-y\left(s \sin \theta-z_{x} s \cos \theta\right)+x_{0} \\
& k=x\left(s \sin \theta+z_{y} s \cos \theta\right)+y\left(s \cdot \cos \theta-z_{y} s \sin \theta\right)+y_{0}
\end{aligned}
$$

Taking the Fourier transform of both sides of (1) and using the linearity, shifting and rotation theorems gives:

$$
\begin{aligned}
F_{2}(u, v) & =e^{-i\left(u x_{0}+v y_{0}\right)} \\
& \times F_{1}\left\{\begin{array}{c}
s(u \cos \theta-v \sin \theta)+s \cdot z_{x}(u \sin \theta+v \cos \theta), \\
s(u \sin \theta+v \cos \theta)+s \cdot z_{y}(u \cos \theta-v \sin \theta)
\end{array}\right\}
\end{aligned}
$$

Taking the magnitude of (3) such that $G_{\mathrm{i}}=\left|F_{\mathrm{i}}\right|$ gives:

$$
G_{2}(u, v)=F_{1}\left\{\begin{array}{c}
s(u \cos \theta-v \sin \theta)+s \cdot z_{x}(u \sin \theta+v \cos \theta), \\
s(u \sin \theta+v \cos \theta)+s \cdot z_{y}(u \cos \theta-v \sin \theta)
\end{array}\right\}
$$

Denoting the Cartesian coordinates $(u, v)$ on the RHS of (4) in terms of the polar coordinates $(r, \phi)$ by substituting for $u=r \cos$ $\phi$ and $v=r \sin \phi$, gives: 


$$
G_{2}(u, v)=F_{1}\left\{\begin{array}{c}
s(r \cos \phi \cos \theta-r \sin \phi \sin \theta) \\
+s \cdot z_{x}(r \cos \phi \sin \theta+r \sin \phi \cos \theta), \\
s(r \cos \phi \sin \theta+r \sin \phi \cos \theta) \\
+s \cdot z_{y}(r \cos \phi \cos \theta-r \sin \phi \sin \theta)
\end{array}\right\}
$$

Applying the product to sum trigonometric identities to (5) results in:

$$
G_{2}(u, v)=F_{1}\left\{\begin{array}{c}
s \cdot r \cos (\theta+\phi)+z_{x} \cdot s \cdot r \sin (\theta+\phi), \\
s \cdot r \sin (\theta+\phi)+z_{y} \cdot s \cdot r \cos (\theta+\phi)
\end{array}\right\}
$$

Mapping expression (6) into the polar domain $(r, \phi)$ where $\boldsymbol{r}=$ $\sqrt{\mathrm{u}^{2}+\mathrm{v}^{2}}$ and $\phi=\arctan (v / u)$ results in:

$$
G_{2}(r, \phi)=G_{1}\left(r \cdot \frac{s}{4} \cdot \sqrt{\beta}, \phi+\tau\right)
$$

where

$$
\beta=H \cdot \sin (K)+T
$$

$$
\begin{aligned}
& H=\left(z_{x}+z_{y}\right) \sqrt{16+\left(z_{y}-z_{x}\right)^{2}} \\
& K=2 \theta+2 \phi+\arctan \left(\frac{z_{y}-z_{x}}{4}\right) \\
& T=2+z_{y}^{2}+z_{x}^{2}
\end{aligned}
$$

and

$$
\tau=\arctan \left(\frac{\tan (\theta+\phi)+z_{y}}{1+z_{x} \tan (\theta+\phi)}\right)-\phi
$$

Taking the logarithm of the first variable in the expression in (7) converts the multiplications into additions to give:

$$
G_{2}(\ln [r], \phi)=G_{1}\left(\ln [r]+\ln [s / 4]+\frac{1}{2} \ln [\beta], \phi+\tau\right)
$$

Now let $\rho=\ln [r], k=\ln [s / 4] b=1 / 2 \ln [\beta]$ and relabeling these relationships as $h_{2}(\omega, \phi)=G_{2}(\rho, \phi)$ and $h_{1}(\omega, \phi)=G_{1}(\rho+k+b$, $\phi+\tau)$ gives the Fourier transform of $h_{2}$ as:

$$
\begin{gathered}
\mathcal{F}\left\{h_{2}(\omega, \phi)\right\}=H_{2}(\varepsilon, \alpha) \\
=\int_{0}^{2 \pi} \int_{0}^{\infty} G_{1}(\omega, \phi) \cdot e^{-i 2 \pi(\varepsilon(\omega+k)+\alpha(\theta+\tau))} \\
\quad \times e^{-i 2 \pi \cdot \varepsilon \cdot b} d \omega d \phi
\end{gathered}
$$

Which can be simplified to:

$$
H_{2}(\varepsilon, \alpha)=H_{1}(\varepsilon, \alpha) \cdot e^{-i(\varepsilon k+\alpha \tau)} \int_{0}^{2 \pi} e^{-i 2 \pi \cdot(\varepsilon \cdot b+\alpha \phi)} d \phi
$$

Substituting for the series expansion of $b$ into the integral in (13) results in it having the approximate form of an $n^{\text {th }}$ order Bessel function of the first kind, denoted as $J_{n}(m)$, where $\mathrm{n}=1$ and $m=2 \pi \varepsilon H$. Accordingly we rewrite (13) as follows:

$$
H_{2}(\varepsilon, \alpha)=H_{1}(\varepsilon, \alpha) \cdot e^{-i(\varepsilon k+\alpha \tau)} \cdot J_{n}(m)
$$

Taking the normalized cross power spectrum of (14) factors out the phase difference since the magnitude of a complex exponential is simply the radius of a unit circle:

$$
\frac{H_{1}(u, v) \cdot H_{2}^{*}(u, v)}{\left|H_{1}(u, v) \cdot H_{2}^{*}(u, v)\right|}=e^{-i(\varepsilon k+\alpha \tau)} \cdot J_{n}(m)
$$

Finally, the Fourier transform of a Bessel function of the first kind is a ring delta function. Convolving it with a Dirac delta at $(k, \tau)$ shifts the ring's origin, so taking the inverse Fourier transform of (15) gives:

$$
\mathcal{F}^{-1}\left\{e^{-i(\varepsilon k+\alpha \tau)} \cdot J_{n}(m)\right\}=\delta(\varepsilon+k, \alpha+\tau) * \operatorname{Ring}(m)
$$

Now since $\varepsilon$ and $\alpha$ are respectively the transformed logarithms of the global scale factor $s$ and the rotation angle $\theta$ we can in theory directly estimate the global scale and rotation from the location of the ring delta on the correlation surface. This is complicated by the fact that the shearing transform partly behaves like rotation and translates the location of the ring based on the direction of the shearing. The shearing magnitude is also not directly related to the ring diameter since the diameter also changes based on the orientation of the shear. If $-z_{x}=z_{y}$ the shearing transform gives pure rotation, as the ring diameter reduces to zero as equations (8) defining the parameters of the Bessel function and (10) defining its location on the rotation axis respectively become:

$$
\begin{gathered}
\beta=2+2 z_{y}^{2} \\
\tau=\theta+\phi+\arctan \left(z_{y}\right)
\end{gathered}
$$

In all other cases the magnitude of the ring is defined by $\sqrt{\beta}$ which as a first order approximation is of the form $\left|z_{y}+z_{x}\right|$.

Another problem is that directly separating the horizontal and vertical components of the shear transform is not feasible from this formulation as the rotation axis $\phi$ becomes nonlinearly warped by equation (10). The eccentricity of $\tau$ increases in proportion to $\sqrt{z_{x}^{2}+z_{y}^{2}}$ and represents compression and expansion along the $\phi$ axis of the form shown in figure 1 . 


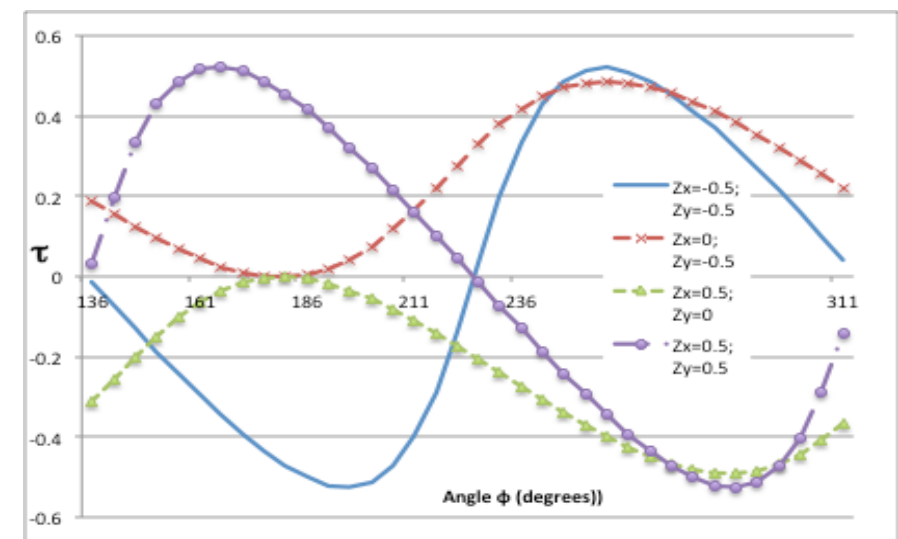

Figure 1. Typical compression and expansion curves along $\phi$ for different values of the $z_{y}, z_{x}$ shearing parameters

\section{METHOD DESCRIPTION}

The first stage in the registration of images that have been subject to shearing is to obtain a translation invariant representation of the images by using the Fourier shift theorem. The resulting images are then remapped using log-polar coordinates to convert rotation and scaling into shifts. Phase registration is then performed using the process described in [2]. The conversion of a image $f(x, y)$ into the log-polar domain $g(\rho, \varphi)$ is performed by resampling the magnitude of the $\mathrm{N} x \mathrm{~N}$ FFTs on an N/2 x N/2 log-polar grid using bilinear interpolation as per:

$$
g(\rho, \varphi)=f\left(N / 2+\beta^{y} \cdot \cos (\alpha), N / 2+\beta^{y} \cdot \sin (\alpha)\right)
$$

Where the variables $\boldsymbol{\alpha}$ and $\boldsymbol{\beta}$ are given by:

$$
\alpha=\frac{\pi \mathrm{x}}{\mathrm{N}} \quad \& \quad \beta=e^{\log (N / 2) / N / 2}
$$

Locating the ring delta on the correlation surface can be performed via a modified Hough Circle Transform [7]. The centroid of the ring $(k, \tau)$ gives the global scaling between the two images, and their rotation shifted by the shearing parameters. The global scaling factor $S$ can be recovered using equations (19) and (20) by first subtracting the ring radius as:

$$
S=N^{1 / N^{k-R}}
$$

The global rotation angle $\theta$ can be recovered from $\tau$ as a first order approximation by again by subtracting the radius of the ring and using the following relationship:

$$
\theta=\frac{\pi \cdot(\tau-R)}{N}
$$

This result incorporates a rotation component introduced by the shearing transform that is reflected in the corresponding estimated shearing parameters.

The radius of the ring $\mathrm{R}$ gives the value of $\sqrt{\beta}$ which can be recovered in a similar manner as (22) where $\mathrm{N}$ is half the number of points in the final Fourier transform:

$$
\sqrt{\beta}=N^{1 / N^{(R+1)}}
$$

If the radius is nonzero additional steps must be taken to estimate the shearing parameters. These can be obtained from the warping that takes place along the $\phi$ axis of the log-polar transformed image expressed by the nonlinearity in $\tau$. The first step is to inverse transform the target image to compensate for any global rotation or scaling that has taken place using the parameters estimated in (21) and (22) such that $\theta=0$ and $s=1$. Each log-polar transformed image is then segmented into a number of moderately narrow bands. Band matching is then used across the two images to find the displacement of the bands in the target image relative to the reference image. Fitting the displacement vectors to equation (10) can be performed using least squares after linearising the relationship as follows:

$Y=z_{y} \cdot X+z_{x}$

Where:

$$
\begin{aligned}
& Y=\tan (\tau+\phi)-\tan (\phi) \\
& X=\tan (\phi) \cdot \tan (\tau+\phi)
\end{aligned}
$$

\section{EXPERIMENTAL RESULTS}

The performance of the proposed scheme was evaluated over a range of global scaling, rotation and shearing parameters using $512 \times 512$ pixel images. These were padded to a size of 1024x1024. Examples of the results are shown in Figures. 2-6. In each of these the transformed image is shown at the top left quadrant, the correlation surface with the detected ring (highlighted) at the top right quadrant and a plot of $\tau$ showing the curvefit for the estimated $z_{x}$ and $z_{y}$ parameters underneath.

The experiments were performed to determine the ability to recover the global scale and rotation of sheared images. Also to determine how the accuracy of the transformation parameter estimation was affected by the orientation and magnitude of the shearing. For this assessment the magnitude $m$ and orientation $\psi$ of the shearing were respectively determined as follows:

$$
m=\sqrt{z_{x}^{2}+z_{y}^{2}} \quad \& \quad \varphi=\arctan \left(\frac{z_{y}}{z_{x}}\right)
$$




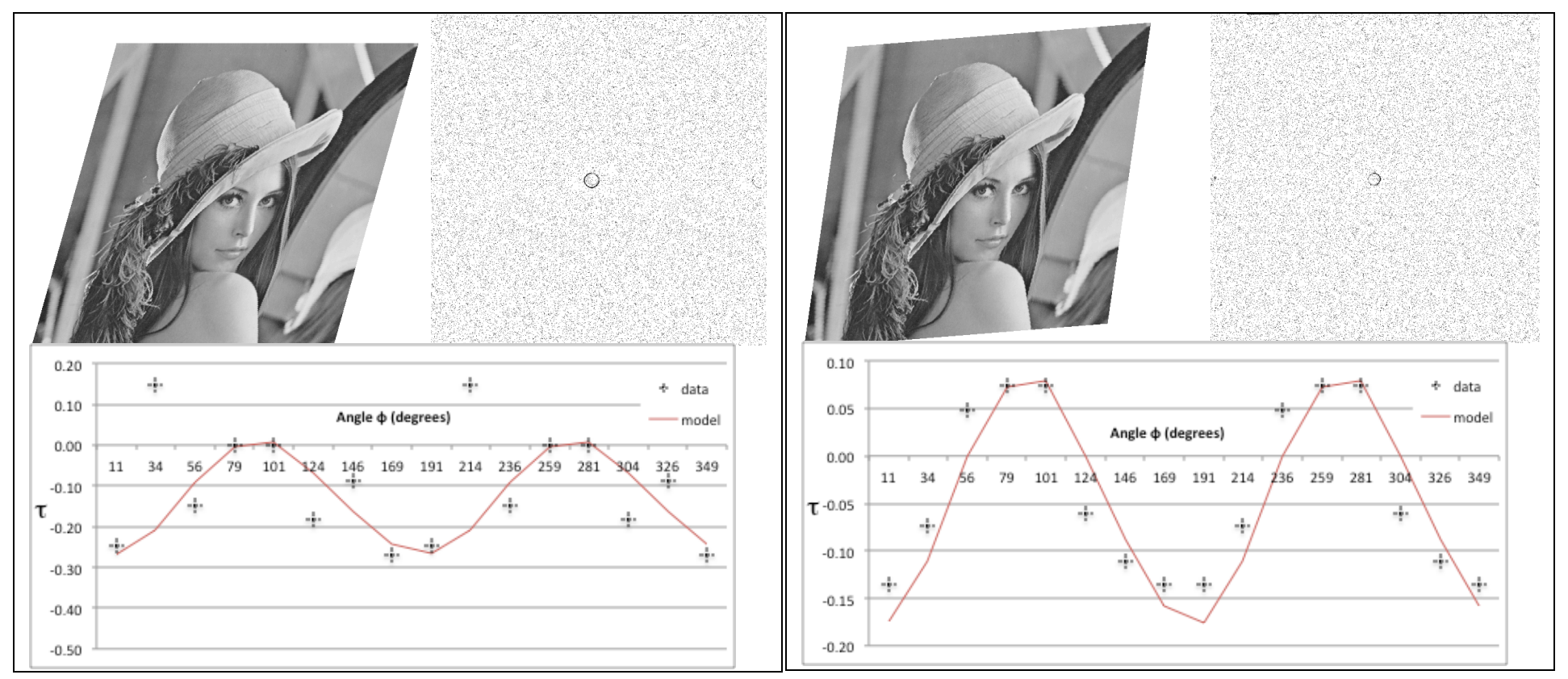

Figure 2. Parameter estimation for image with transform parameters $r=0, s=1.0$, $\mathrm{z}_{\mathrm{x}}=-0.281$ and $\mathrm{Zy}=0$. The ring is centered at $(-10,0)$ with radius $=12$, estimated $z_{x}=-0.249, Z y=-0.008$.

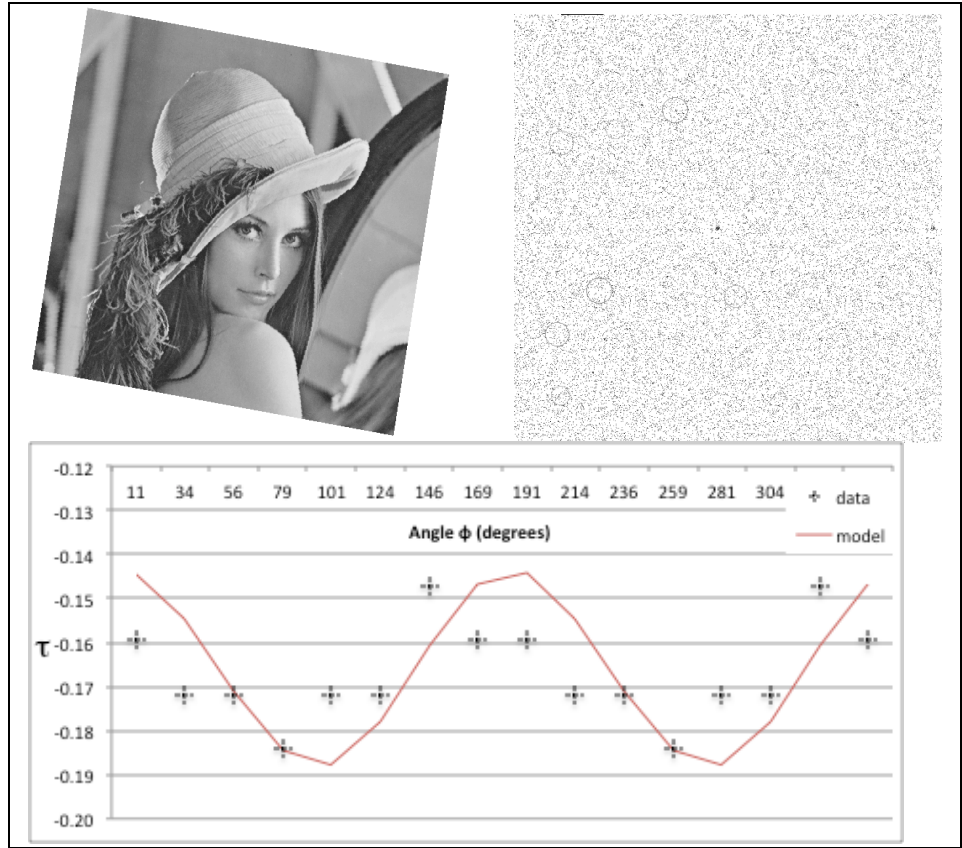

Figure 3. Parameter estimation for image with transform parameters $\mathrm{r}=0, \mathrm{~s}=1.0$, $z_{x}=-0.249, Z y=0.2187$. The ring is centered at $(-14,-1)$ with radius $=2$, estimated $\mathrm{Z}_{\mathrm{x}}=-0.208, \mathrm{Zy}=0.173$
Figure 4. Parameter estimation for image with transform parameters $r=0, s=1.0$, $Z_{x}=-0.15$ and $Z y=-0.094$. The ring is centered at $(-2,0)$ with radius $=10$, estimated $z_{x}=-0.198, Z y=-0.081$.
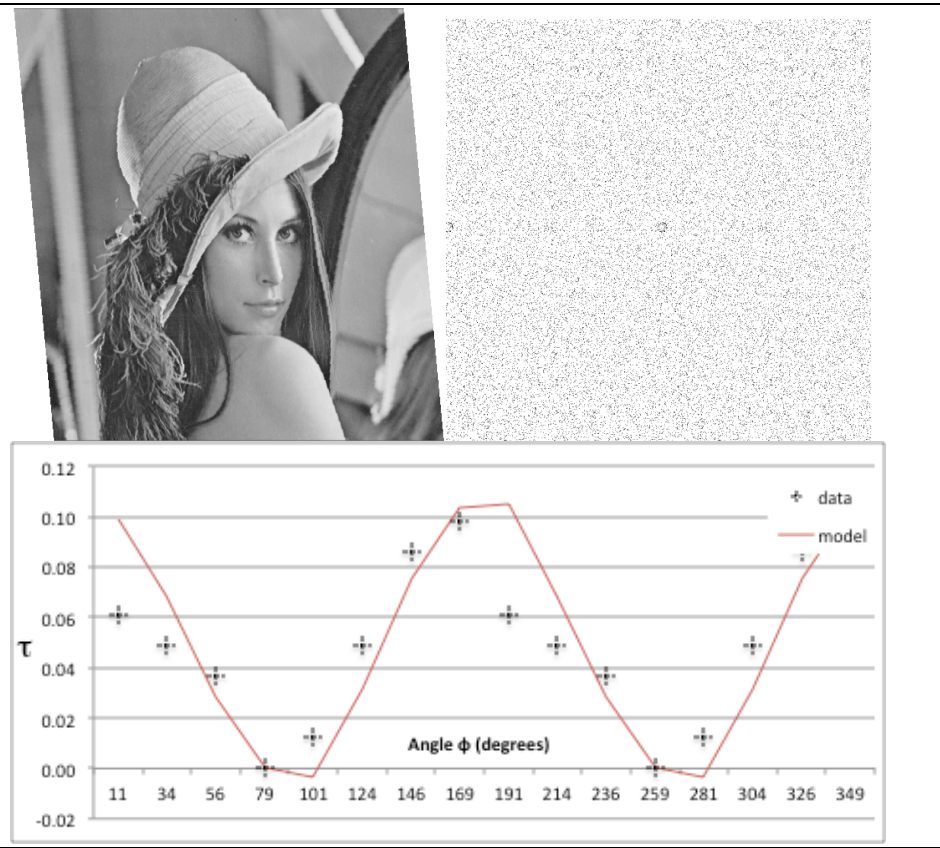

Figure 5. Parameter estimation for image with transform parameters $r=0, s=1.1$, $z_{x}=0.094$ and $Z y=0$. The ring is centered at $(4,2)$ with radius $=6$, estimated $z_{x}=$ $0.096, \mathrm{Zy}=0.003$. 


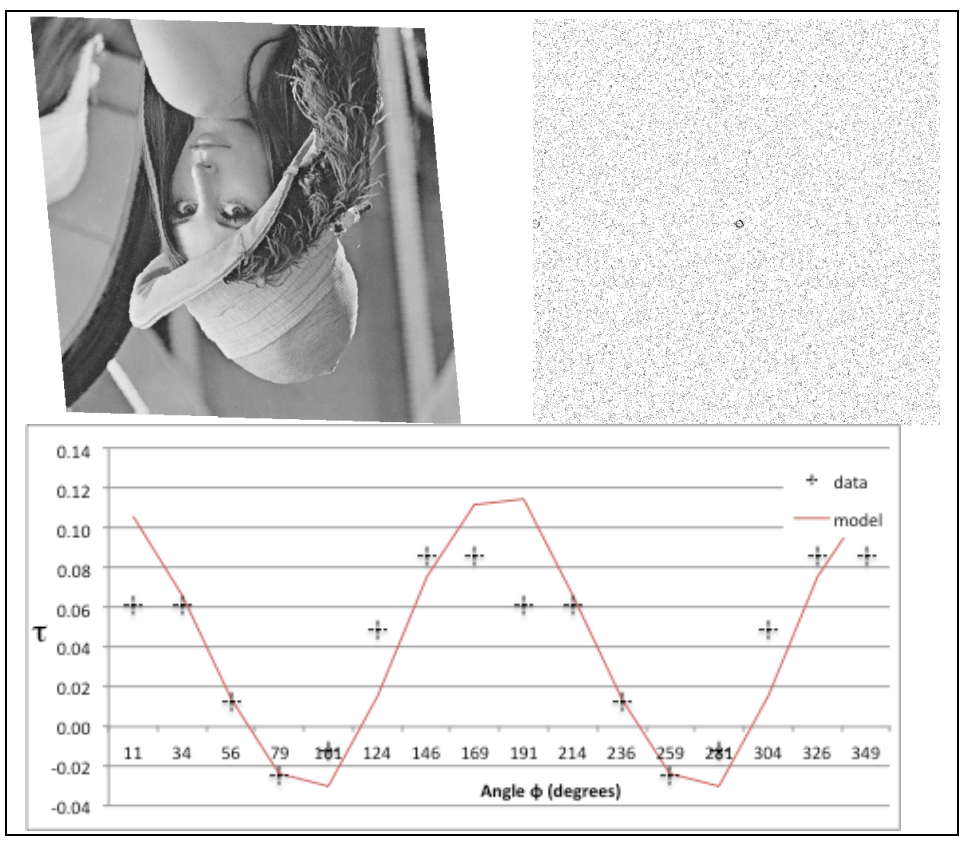

Figure 6. Parameter estimation for image with transform parameters $r=-180$, $\mathrm{s}=1.0, \mathrm{z}_{\mathrm{x}}=0.094$ and $\mathrm{z}_{\mathrm{y}}=0$. The ring is centered at $(-2,0)$ with radius $=5$, estimated $\mathrm{z}_{\mathrm{x}}=0.097, \mathrm{z}_{\mathrm{y}}=0.021$.

The accurate recovery of the global scale and rotation however decreases as the amount of shear increases. Figure 7 plots the scale factors reliably recovered on average for different amounts of horizontal shear as a percent of image size. A given scale factor was deemed to be reliably detected if the average error at a given amount of shear for all rotation angles was below $15 \%$. This error threshold was chosen rather arbitrarily since error distribution tends to be a step function: where the rings are correctly identified the error is typically below $15 \%$, otherwise the average error is above $40 \%$.

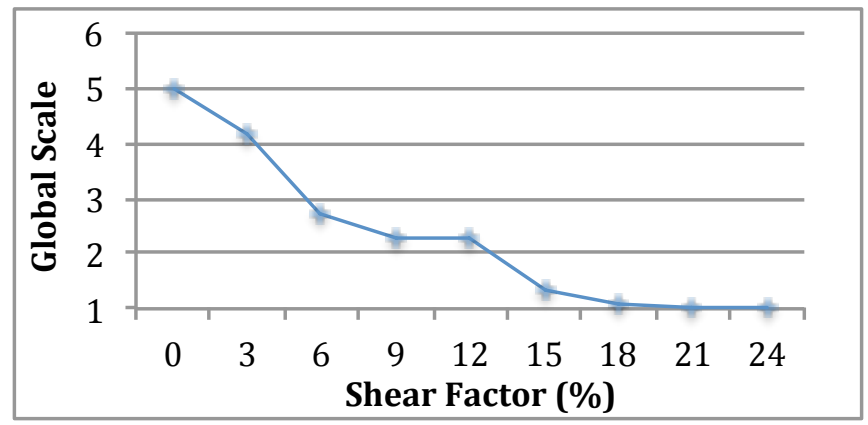

Figure 7. Recovery of scale factors for different amounts of shear.

First the sensitivity of shear parameter estimation to the orientation of the shear was evaluated. The shearing parameters were estimated for a set of image pairs that had undergone shearing of a fixed magnitude (12.5\% of the image size) for orientation values of $0 \leq \psi \leq 360$ in 10 degree increments. The absolute error of the estimated $z_{x}$ and $z_{y}$ relative to the ground truth remained fairly uniform across the entire range of orientations as is shown in Figure 8. The mean absolute errors were $\mathrm{z}_{\mathrm{x}}=0.014, \mathrm{z}_{\mathrm{y}}=0.005$ and respectively correspond to percent median errors of $12.5 \%$ and $3.9 \%$.

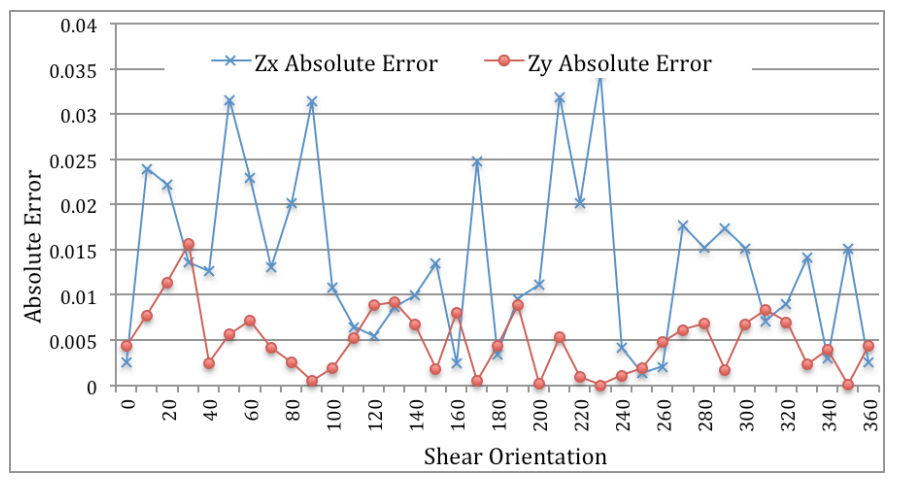

Figure 8. Absolute parameter estimation errors as a function of orientation.

The shearing parameters were then estimated for image pairs that had undergone shearing of magnitudes in the range of $0 \leq m \leq$ $0.625(62.5 \%$ of image size $)$ in steps of 0.03125 for fixed orientations. The absolute error of the estimated $z_{x}$ and $z_{y}$ relative to the ground truth is shown in Figure 9. The error initially falls as the shear magnitude increases to about $20 \%$ due to improving precision in the calculation. It then begins to increase in a linear fashion past magnitudes of around $35 \%$, mainly due to increasing noise on the correlation surface. The error at low shearing magnitudes is due to limited resolution in the log polar transformed images. For shearing magnitudes below $40 \%$ of the image size the mean absolute errors were $\mathrm{z}_{\mathrm{x}}=0.1$ and $\mathrm{z}_{\mathrm{y}}=0.07$. This corresponds to a median percent error of about $19 \%$ for shearing magnitudes in the range of $0.2-0.4$.

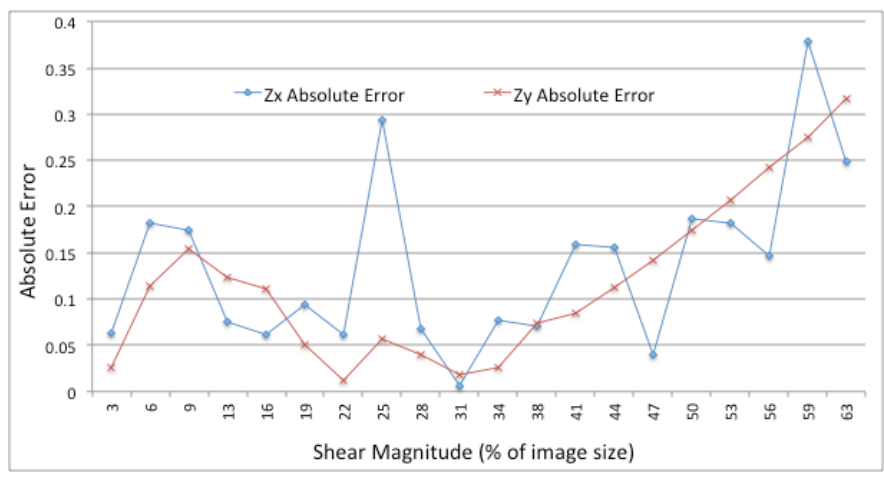

Figure 9. Absolute parameter estimation errors as a function of magnitude.

Without compensating for global scaling the accuracy of the shearing parameter estimation begins to deteriorate as scaling ratios of 1.3 are exceeded. The results in Figure 10 show the accuracy of $z_{x}$ and $z_{y}$ estimates for fixed shearing of $30 \%$ and $25 \%$ (averaged) at different global scaling factors. Below global scale ratios of 1.4 , the median percent error for the $z_{x}$ estimate was $4.3 \%$. For higher scaling ratios, preliminary compensation for the 
global scaling is recommended in order estimate the shearing parameters accurately

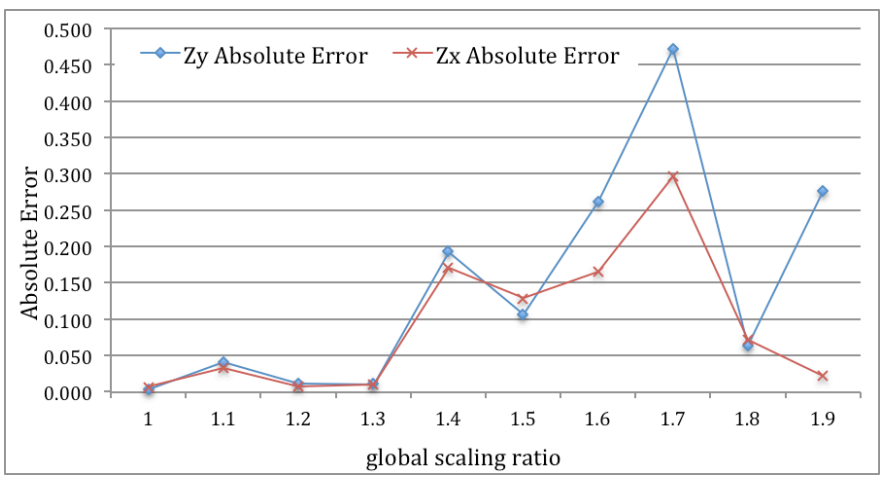

Figure 10. Absolute $\mathrm{z}_{\mathrm{x}} \& \mathrm{z}_{\mathrm{y}}$ estimation errors as a function of global scaling.

Without compensating for global rotation the accuracy of the shearing parameter estimation deteriorates very rapidly when any rotation exceeds \pm 10 degrees. This is to be expected since shearing transformations induce rotation that affects the estimation process. This can be seen in Figure 11 showing the absolute error of $z_{x}$ and $z_{y}$ estimates for fixed shearing of $30 \%$ and $25 \%$ at different scale factors and represent a median error of $45 \%$. Comparing these results to those of fig. 8 reveals the importance of compensating for global rotation prior to the estimation of the shearing parameters.

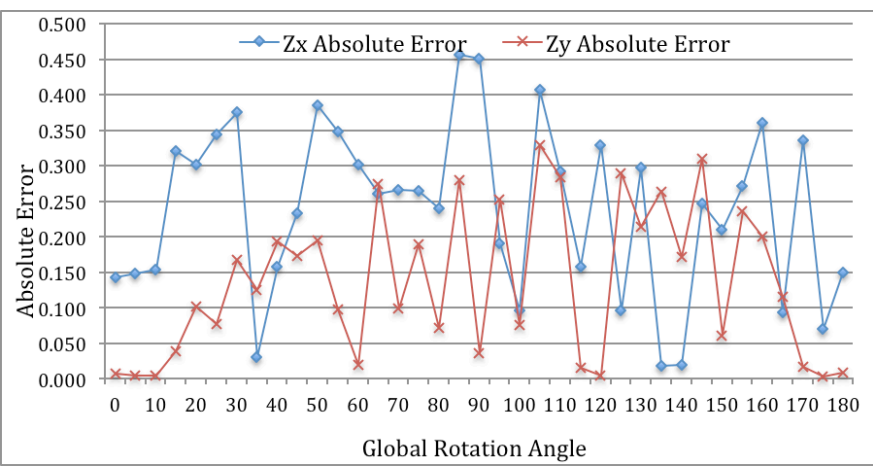

Figure 11. Absolute $\mathrm{z}_{\mathrm{x}} \& \mathrm{z}_{\mathrm{y}}$ estimation errors as a function of global rotation.

Appropriately compensating for any global scaling and rotation enables the reasonably accurate recovery of shearing transformation parameters for shearing transformation of up to $40 \%$ of the image size.

\section{CONCLUSIONS}

This paper has presented a novel, straightforward method for estimating the transformation parameters of images that have undergone global scaling, rotation, translation and arbitrary shearing for image registration. Based on Phase Correlation it provides noise resilience and constant processing time in a closed form solution. While the distortion introduced by shearing limits the upper range of global scaling that could otherwise be recovered, the proposed method is able to recover shearing parameters over all possible shearing orientations with reasonable accuracy for shear magnitudes below $40 \%$ of the image size.

\section{REFERENCES}

[1] B.S. Reddy, B.N. Chatterji, "An FFT-based technique for translation, rotation, and scale-invariant image registration", IEEE Trans. on Image Processing, vol.5, no. 8, pp.1266-1271, 1996.

[2] R. Gonzalez, "Improving Phase Correlation for Image Registration", Proceedings of (ICVNZ2011) Image and Vision Computing New Zealand 2011, p.488-493, Nov.29 - Dec.1., http://www.ivs.auckland.ac.nz/ivenz2011/proceedings.pdf

[3] S. Zokai, G. Wolberg, "Image Registration Using Log-Polar Mappings for Recovery of Large-Scale Similarity and Projective Transformations." IEEE Trans. Image Processing, vol.14, no.10, pp.1422-1434, 2005

[4] B.J. Super, A.C. Bovik, "Shape from texture using local spectral moments”, IEEE Trans. PAMI Apr 1995 Vol.17, No. 4 pp 333 - 343

[5] J. Krumm, "Shape from periodic texture using the spectrogram", Computer Vision and Pattern Recognition, 1992. Proceedings CVPR '92, 15-18 Jun 1992, pp 284 - 289

[6] L. Lucchese, S. Leorin, GM. Cortelazzo, "Estimation of twodimensional affine transformations through polar curve matching and its application to image mosaicking and remote-sensing data registration." IEEE Trans. Image Process. 2006 Oct; Vo.15, No. 10, pp.3008-19.

[7] R. Gonzalez "Robust Ring Detection in Phase Correlation Surfaces" to appear DICTA2013 